\title{
Predictors of symptomatic lymphocele after kidney transplantation
}

\author{
Maja Joosten ${ }^{1}$ (1) $\cdot$ Frank C. d'Ancona ${ }^{2} \cdot$ Wilbert A. van der Meijden $^{3} \cdot$ Paul P. Poyck $^{1}$
}

Received: 3 June 2019 / Accepted: 28 August 2019 / Published online: 5 September 2019

(c) The Author(s) 2019

\begin{abstract}
Purpose The development of a symptomatic lymphocele (SL) is a frequent postoperative surgical complication after kidney transplantation. It may lead to pain and discomfort and cause transplant malfunction or even secondary graft loss. A large cohort of renal recipients was investigated to identify the possible risk factors for SL.

Methods All renal transplant patients of a single centre were retrospectively analysed for SL between January 2010 and December 2017. The SL group was compared to a control group from the same cohort.

Results 45 out of 1003 transplanted patients developed an SL (incidence 4.5\%), on average 50 days after kidney transplantation. SLs developed more in older patients, in those with a PD catheter and in ADKDP as primary diagnosis. Surgical predictors for SLs were venous anastomosis on the external iliac vein, concomitant PD catheter removal, perfusion defects, shorter operating time, splint $>7$ days, double J stenting, discharge with drain, low initial drain production and ureteral obstruction. Opening of the peritoneum, re-operation for postoperative bleeding and previous nephrectomy seem protective for developing SL.

Conclusion We found multiple heterogeneous predictors for SL with a common denominator related to surgical management of the retroperitoneal space, peritoneum and the ureter. Future prospective studies are necessary to evaluate the influence of these variables on the development of SL.
\end{abstract}

Keywords Renal transplantation $\cdot$ Complication $\cdot$ Lymphocele $\cdot$ Risk factors $\cdot$ Predictors

\section{Abbreviations}

ADPKD Autosomal dominant polycystic kidney disease

Ao Aorta

BMI Body mass index

CI Confidence interval

CIA Common iliac artery

CIT Cold ischaemia time

CIV Common iliac vein

EIA External iliac artery

EIV External iliac vein

ESRD End-stage renal disease

IVC Inferior vena cava

Paul P. Poyck

paul.poyck@radboudumc.nl

1 Department of Vascular and Transplant Surgery, Radboudumc, Postbus 9101, 6500 HB Nijmegen, The Netherlands

2 Department of Urology, Radboudumc, Nijmegen, The Netherlands

3 Department of Nephrology, Radboudumc, Nijmegen, The Netherlands
PD Peritoneal dialysis

SL Symptomatic lymphocele

TUC Trans-urethral catheter

\section{Introduction}

One of the most frequently observed postoperative complications of kidney transplantation is a symptomatic perirenal fluid collection, such as a haematoma, a lymphocele or lymphorrhea. A lymphocele in renal transplant recipients is a pseudo-cystic entity filled with lymph fluid, covered with a hard fibrous capsule, localized around the graft. The incidence of lymphoceles after kidney transplantation varies widely and is reported between $0.03 \%$ and $33.9 \%$ with a mean incidence of 5.2\% [1-8]. Lymphoceles are diagnosed primarily by sonographic imaging and occur from 2 weeks up to 6 months after transplantation with a peak incidence at 6 weeks [9].

Most lymphoceles are asymptomatic and resolve spontaneously or even go unnoticed. Symptomatic lymphoceles (SLs), however, cause pain and discomfort mainly due to 
the presence of a mass in the lower abdominal quadrant. More severe manifestations are ureteral obstruction, infection or renal vein thrombosis that may lead to secondary graft deterioration or even graft loss. Treatment consists of (repeated) aspiration or drainage of fluid, or more invasively, by open or laparoscopic intraperitoneal marsupialization or fenestration, which in turn may lead to complications as well [10-12]. Despite a variety of treatment options, symptomatic lymphoceles remain difficult to treat and frequently lead to recurrent hospital admissions. This has a large impact on the physical and mental wellbeing of the patient as well as on community or personal financial resources.

Current research has mainly focused on the treatment of choice for lymphoceles. There is no consensus about the risk factors for the development of an SL or how to prevent symptomatic lymphoceles from developing [13-15]. We, therefore, retrospectively investigated a large cohort of renal recipients to identify factors that can predict the development of a symptomatic lymphocele and may be possible risk factors.

We hypothesized that risk factors could be divided into three categories: patient characteristics (e.g. a higher BMI, previous abdominal surgery or a previous kidney transplantation in the same fossa, smoking, atherosclerosis), surgical characteristics (e.g. longer surgery time, more dissection needed) and postoperative characteristics (e.g. need for a splint longer than 7 days, need for a nephrostomy catheter).

\section{Materials and methods}

\section{Study design and study population}

For this retrospective, observational, cohort study, the electronic medical records of all 1003 renal transplant recipients from a single academic centre (Radboudumc, Nijmegen, The Netherlands) were reviewed for the development of a symptomatic lymphocele between January 2010 and December 2017. Symptomatic lymphocele was defined as a symptomatic fluid collection near the graft that required an intervention for the graft or patient. Postoperative or postintervention haematomas, urinomas and abscesses were excluded. Since lymphoceles mostly develop within 6 months after renal transplantation, patients needed to have a follow-up time of at least 6 months.

The control group consisted of 520 patients who were randomly selected from the same cohort and evenly distributed over the cohort period from the beginning of 2014 until the end of 2017 (due to the use of an electronic patient system since 2014). According to regulation, no separate approval of the Medical Center Ethical Review Committee was necessary. Informed consent was waived.

\section{Kidney transplantation}

All operations were performed by a senior surgeon sufficiently trained for kidney transplantation. All recipients were given prophylactic broad-spectrum antibiotics $(2 \mathrm{~g}$ ceftriaxone iv) $30 \mathrm{~min}$ prior to incision.

A retroperitoneal approach via a Gibson incision was routinely used for the arterial and venous anastomoses onto the iliac vessels and to perform a ureteroneocystostomy according to Lich-Gregoir subsequently.

If applicable a PD catheter was surgically removed at the end of the procedure. The ureteroneocystostomy was stented using an external splint for 5-7 days. To shorten the hospital admission time, from 2017 a double $\mathrm{J}$ stent was inserted and removed 2-3 weeks later at the outpatient clinic. At the end of the procedure, a wound drain was placed just beside the ureteroneocystostomy.

\section{Immunosuppressive therapy}

All patients received triple immunosuppressive therapy consisting of tacrolimus (trough level 8-12 $\mu \mathrm{g} / \mathrm{l}$ ), mycophenolic acid and prednisolone. After 6 months, tacrolimus (trough level 5-8 $\mu \mathrm{g} / \mathrm{l})$, prednisolone $(0.1 \mathrm{mg} /$ $\mathrm{kg} /$ day) and mycophenolic acid dose was decreased and then discontinued, unless the patient had side effects of prednisolone. In these cases, prednisolone was decreased and stopped instead of mycophenolic acid. No induction therapy was given before July 2014, whereas thereafter basiliximab was given as induction immunosuppressant. If patients developed a symptomatic lymphocele, the immunosuppressive therapy was not adjusted.

\section{Study variables}

To assess risk factors for development of a symptomatic lymphocele, study variables were extracted from the electronic patient record. The study variables in this retrospective study were divided into patient characteristics, surgery related and related to the postoperative period. Patient characteristics were gender, age at day of transplantation, weight, body mass index, primary diagnosis for renal failure, cardiovascular history, smoking, diabetes mellitus, hypertension, kidney replacement therapy, type of kidney replacement therapy, duration of peritoneal dialysis previous to transplantation, PD catheter at time of surgery, side of the PD catheter, previous abdominal surgery, previous native nephrectomy (ADKDP patients), radiotherapy of pelvis, previous transplantation in same fossa, total kidney transplants, type of donor (living/deceased), 
$\mathrm{AB} 0$ incompatible donor, and need for anti-T cell therapy in case of rejection.

Surgical characteristics were fossa sidedness, atherosclerosis of the iliac arteries, reconstruction of the iliac arteries, cold ischaemia time, anastomosis time, total surgery time, total blood loss, time on ventilation, whether the peritoneum was opened, removal and side of PD catheter, visible perfusion defects after reperfusion, diuresis and placement of splint or double J. Furthermore, for vascular anatomy of the graft and location of the anastomosis, high was defined as an arterial anastomosis on the common iliac artery or aorta, low arterial anastomosis was defined as an anastomosis on the external iliac artery and high venous anastomosis defined as an anastomosis on the common iliac vein and inferior vena cava and low venous anastomosis was defined as an anastomosis on the external iliac vein.

Postoperative characteristics were defined as postoperative bleeding requiring re-operation, drain production and drain in situ at discharge, re-insertion of a drain, marsupialization or fenestration, splint in situ more than 7 days, transurethral catheter in situ for more than 9 days, ureteral obstruction, placement of nephrostomy catheter, any intervention done and mortality.

\section{Statistical analysis}

Univariate analysis was performed on all individual study variables using an unpaired $t$ test to identify variables that were predominantly present in either the SL- or the control group, therefore being possible risk factors for the development of an SL. Data are expressed as mean \pm standard deviation for continuous variables and as numbers and percentage for non-metric parameters. A $P$ value $<0.05$ was considered statistically significant. Statistical analysis was performed using statistical package for social sciences (SPSS) version 25.

\section{Results}

Between January 2010 and December 2017, 1003 patients received a kidney transplantation and 45 patients developed a symptomatic lymphocele. The overall incidence of SLs in this study group was $4.5 \%$ (95\% CI 3.6-5.8\%). Incidence per year varied between $1.0 \%$ and $11.0 \%$. SLs were detected after an average of 50 days post-surgery (range 11-244 days); this was on average 43 days after the first ultrasound.

Patients in the SL group had a higher age in comparison with the control group $(56 \pm 13$ vs. $47 \pm 17$ years, $P=0.017)$, more patients had a PD catheter at the moment of transplantation $(22 \%$ vs. $18 \%, P=0.007)$ and more patients in the SL group were diagnosed with ADPKD (31\% vs. 16\%, $P=0.001$ ).

In the SL group, there were fewer patients with ADPKD that previously had a nephrectomy $(0 \%$ vs. $10 \%, P=0.030)$ and less patients with a PD catheter at the same side as the kidney transplantation ( $0 \%$ vs. $36 \%, P=0.000$ ). No significant differences were found in all other patient characteristics (Table 1).

Comparing the SL group to the control group, more SLs were seen in patients that had a low venous anastomosis ( $11 \%$ vs. $5.4 \%, P=0.021$ ), removal of the PD catheter at the end of the kidney transplantation ( $18 \%$ vs. $14 \%, P=0.014$ ), perfusion defects of the kidney after completion of the anastomosis and reperfusion $(20 \%$ vs. $7.3 \%, P=0.000)$, and shorter surgery time $(156 \pm 33$ vs. $165 \pm 41 \mathrm{~min}, P=0.012)$.

In the SL group, there was less removal of the PD catheter located at the same side as the kidney graft $(0 \%$ vs. $25 \%$, $P=0.006)$ and less opening of the peritoneum (0\% vs. $6.4 \%$, $P=0.000)$. No differences were found in all other surgical items (Table 1).

In the SL group, there were more patients that needed a splint for more than seven days ( $2.2 \%$ vs. $0.6 \%, P=0.009)$, more usage of double $\mathrm{J}$ catheters ( $22 \%$ vs. $11 \%, P=0.000$ ), more drain requirement at discharge $(6.7 \%$ vs. $1.3 \%$, $P=0.000)$ and more ureteral obstructions (18\% vs. $4.2 \%$, $P=0.000)$.

In contrast to this, there was less production of the postoperative drain in comparison with the control group $(147 \pm 188 \mathrm{ml}$ vs. $359 \pm 533 \mathrm{ml}, P=0.011)$ and less patients that needed re-operation for postoperative bleeding ( $0 \%$ vs. $2.5 \%, P=0.03)$. No significant differences were found in the other postoperative parameters (Table 1).

\section{Discussion}

Symptomatic lymphoceles are frequently reported surgical complications after kidney transplantation and may cause devastating harm to the transplant as well as the patient. Since we noticed a peak in the incidence of SLs in our hospital, we investigated our complete cohort over the last 7 years. The overall incidence was $4.5 \%$ (range $1.0-11 \%$ ), which is below the mean $5.2 \%$ described in literature [1-8]. The most important finding of this study was the heterogenicity of the predictors. In other words, multiple heterogeneous surgical and non-surgical predictors were related to SL, although a common denominator seems to relate to the management of the retroperitoneal space, peritoneum and the ureter. Furthermore, we found an overall mortality that was sixfold higher in the SL group. Causes of death of the transplant recipients were colon carcinoma (1144 days and 1441 days after kidney transplantation), Epstein-Barr virus-driven post-transplant 
Table 1 Patient, surgical and postoperative variables

\begin{tabular}{|c|c|c|c|}
\hline & Control group & SL group & $P$ value \\
\hline \multicolumn{4}{|l|}{ Patient variables } \\
\hline Male & $58 \% ; 302 / 520$ & $60 \% ; 27 / 45$ & 0.539 \\
\hline Mean age at kidney transplant (years) & $47 \pm 17$ & $56 \pm 13$ & 0.017 \\
\hline Mean weight (kg) & $75 \pm 19$ & $78 \pm 18$ & 0.277 \\
\hline Mean body mass index & $25 \pm 4.8$ & $26 \pm 6.4$ & 0.861 \\
\hline Second or more transplants & $19 \%, 101 / 520$ & $27 \% ; 12 / 45$ & 0.340 \\
\hline AB0 incompatible donor & $2.9 \% ; 15 / 520$ & $4,4 \% ; 2 / 45$ & 0.627 \\
\hline Anti T-cell therapy & $8.5 \% ; 44 / 520$ & $13 \% ; 6 / 45$ & 0.179 \\
\hline Kidney replacement therapy & $76 \% ; 393 / 520$ & $76 \% ; 34 / 45$ & 0.924 \\
\hline Peritoneal dialysis & $19 \% ; 96 / 520$ & $22 \% ; 10 / 45$ & 0.565 \\
\hline Duration of peritoneal dialysis (days) & $867 \pm 599$ & $862 \pm 478$ & 0.979 \\
\hline Haemodialysis & $57 \% ; 297 / 520$ & $53 \% ; 24 / 45$ & 0.659 \\
\hline Previous kidney transplant in same fossa & $4.6 \% ; 24 / 520$ & $2.2 \% ; 1 / 45$ & 0.147 \\
\hline Previous abdominal surgery & $41 \% ; 211 / 520$ & $44 \% ; 20 / 45$ & 0.337 \\
\hline Previous nephrectomy (ADKDP) & $10 \% ; 52 / 520$ & $0 \% ; 0 / 45$ & 0.030 \\
\hline Radiotherapy of pelvic region & $1.5 \% ; 8 / 520$ & $2.2 \% ; 1 / 45$ & 0.450 \\
\hline Pre-transplant cardiovascular disease & $24 \% ; 127 / 520$ & $22 \% ; 10 / 45$ & 0.760 \\
\hline Pre-transplant smoking & $17 \% ; 87 / 520$ & $8.9 \% ; 4 / 45$ & 0.120 \\
\hline Pre-transplant diabetes mellitus & $14 \% ; 72 / 520$ & $13 \% ; 6 / 45$ & 0.684 \\
\hline Pre-transplant hypertension & $47 \% ; 243 / 520$ & $42 \% ; 19 / 45$ & 0.110 \\
\hline Donor type: living & $64 \% ; 334 / 520$ & $62 \% ; 28 / 45$ & 0.115 \\
\hline Donor type: deceased & $36 \% ; 186 / 520$ & $38 \% ; 17 / 45$ & 0.499 \\
\hline PD catheter at time of kidney transplant & $18 \% ; 96 / 520$ & $22 \% ; 10 / 45$ & 0.007 \\
\hline PD catheter at same side as kidney transplant & $36 \% ; 35 / 96$ & $0 \% ; 0 / 10$ & 0.000 \\
\hline \multicolumn{4}{|l|}{ Surgical variables } \\
\hline Artery anatomy: single & $76 \% ; 396 / 520$ & $69 \% ; 31 / 45$ & 0.319 \\
\hline Artery anatomy: multiple & $24 \% ; 124 / 520$ & $31 \% ; 14 / 45$ & 0.319 \\
\hline Sacrifice of renal arteries & $7.1 \% ; 37 / 520$ & $11 \% ; 5 / 45$ & 0.340 \\
\hline Right fossa & $74 \% ; 385 / 520$ & $76 \% ; 34 / 45$ & 0.998 \\
\hline Low arterial anastomosis (EIA) & $33 \% ; 173 / 520$ & $36 \% ; 16 / 45$ & 0.761 \\
\hline High arterial anastomosis (AO and CIA) & $67 \% ; 347 / 520$ & $64 \% ; 29 / 45$ & 0.761 \\
\hline Low venous anastomosis (EIV) & $5.4 \% ; 28 / 520$ & $11 \% ; 5 / 45$ & 0.021 \\
\hline High venous anastomosis (IVC and CIV) & $95 \% ; 492 / 520$ & $89 \% ; 40 / 45$ & 0.021 \\
\hline Mean CIT living donor (min) & $127 \pm 34$ & $118 \pm 39$ & 0.753 \\
\hline Mean CIT deceased donor (min) & $771 \pm 286$ & $864 \pm 260$ & 0.878 \\
\hline Mean anastomosis time (min) & $25 \pm 10$ & $25 \pm 12$ & 0.141 \\
\hline Mean total surgery time (min) & $165 \pm 41$ & $156 \pm 33$ & 0.012 \\
\hline Mean total ventilation time (min) & $195 \pm 61$ & $188 \pm 40$ & 0.626 \\
\hline Peritoneum opened & $6.4 \% ; 33 / 520$ & $0.0 \% ; 0 / 45$ & 0.000 \\
\hline Removal PD catheter & $14 \% ; 73 / 520$ & $18 \% ; 8 / 45$ & 0.014 \\
\hline Removed PD catheter at same side as kidney transplant & $25 \% ; 18 / 73$ & $0 \% ; 0 / 8$ & 0.006 \\
\hline Perfusion defect after anastomosis & $7.3 \% ; 38 / 520$ & $20 \% ; 9 / 45$ & 0.000 \\
\hline Diuresis on table & $91 \% ; 474 / 520$ & $84 \% ; 38 / 45$ & 0.510 \\
\hline Atherosclerosis (seen during surgery) & $11 \% ; 59 / 520$ & $6.7 \% ; 3 / 45$ & 0.610 \\
\hline Reconstruction iliac arteries & $1.7 \% ; 9 / 520$ & $0.0 \% ; 0 / 45$ & 0.790 \\
\hline Mean total blood loss (ml) & $372 \mathrm{ml} \pm 383 \mathrm{ml}$ & $317 \mathrm{ml} \pm 304 \mathrm{ml}$ & 0.610 \\
\hline Double J & $11 \% ; 57 / 520$ & $22 \% ; 10 / 45$ & 0.000 \\
\hline \multicolumn{4}{|l|}{ Postoperative variables } \\
\hline Drain production $(\mathrm{ml})$ & $359 \pm 533$ & $147 \pm 188$ & 0.011 \\
\hline TUC for more than 9 days & $1.5 \% ; 8 / 520$ & $0.0 \% ; 0 / 45$ & 0.099 \\
\hline Splint for more than 7 days & $0.6 \% ; 3 / 520$ & $2.2 \% ; 1 / 45$ & 0.009 \\
\hline
\end{tabular}


Table 1 (continued)

\begin{tabular}{llll}
\hline & Control group & SL group & $P$ value \\
\hline Nephrostomy catheter & $2.9 \% ; 12 / 520$ & $2.2 \% ; 1 / 45$ & 0.906 \\
Drain requirement at discharge & $1.3 \% ; 7 / 520$ & $6.7 \% ; 3 / 45$ & 0.000 \\
Surgery for postoperative bleeding & $2.5 \% ; 13 / 520$ & $0.0 \% ; 0 / 45$ & 0.033 \\
Marsupialisation & $0.2 \% ; 1 / 520$ & $20 \% ; 9 / 45$ & 0.000 \\
Drain re-insertion & $1.3 \% ; 7 / 520$ & $100 \% ; 45 / 45$ & 0.000 \\
Ureteral obstruction & $4.2 \% ; 22 / 520$ & $18 \% ; 8 / 45$ & 0.000 \\
Intervention done & $6.7 \% ; 35 / 520$ & $100 \% ; 45 / 45$ & 0.001 \\
Deceased & $1.5 \% ; 8 / 520$ & $8.9 \% ; 4 / 45$ & 0.000 \\
\hline
\end{tabular}

Data are expressed as the mean \pm standard deviation, percentages or number of patients per cohort

Ao aorta, CIA common iliac artery, EIA external iliac artery, EIV external iliac vein, IVC inferior vena cava, $C I V$ common iliac vein, CIT cold ischaemia time, TUC trans-urethral catheter

lymphoproliferative disease (309 days after kidney transplantation), metastatic squamous cell carcinoma of the lung (295 days after kidney transplantation), respiratory insufficiency due to pneumonia (154 and 200 days after kidney transplantation) and metastatic cancer with unknown primary tumour in the control group (665 days and 1354 days after kidney transplantation). In the SL group, the causes were diffuse large B cell cerebral lymphoma (161 days after kidney transplantation), sepsis due to perforation of the colon (764 days after kidney transplantation), infected ascites (2153 days after kidney transplantation) and metastatic cholangiocarcinoma (1606 days after kidney transplantation). Due to the large number of days between kidney transplantation and deceasing (range 154-2153 days), there seems to be no correlation between the two. We are, therefore, unable to explain the higher mortality in the SL group. At the time of kidney transplantation, no patients were known to have a (metastatic) carcinoma. A malignancy was a contra-indication for kidney transplantation. Despite the fact that causes of death did not seem to be directly related to an SL, this finding should encourage further research and awareness in the management of kidney transplant patients. We will further discuss the other predictors for SL that this study has found.

A predictable but not less important patient-related factor in the patient characteristics was age. Older patients receiving a kidney transplant were more at risk of developing an SL. Since more patients are treated at higher age, this effect should be addressed to the patient in light of the need for more invasive treatment and higher mortality risk if an SL occurs. In the literature, age as a predictor for lymphoceles has previously been described for prostatectomy. Older patients are prone to a decreased nutritional status accompanied by hypo-albuminemia and hypo-proteinemia, resulting in impaired tissue healing and prolonged lymphorrhea [16].

High body mass index has been described in the literature to increase the risk of SL [17]. We were not able to establish the same effect in this study.
Autosomal dominant polycystic kidney disease (ADPKD) was the only end-stage renal disease (ESRD) that led to an increase of SLs in this study. Martinez-Ocana et al. also reported this correlation [18]. They suggested that the enlargement of the native kidneys could compress the inferior vena cava, which would result in reduction of the lymphatic flow. This in turn would lead to more lymph fluid around the graft, possibly leading to more lymphoceles [18, 19]. Interestingly, we also found a supporting averse effect since a nephrectomy of a large cystic kidney prior to transplantation reduced the risk of SLs.

One of the surgical parameters that was found to increase the risk of an SL was the use of a low venous anastomosis. This effect has previously been described by Sansalone et al.; they pointed out that dissection around iliac vessels should be done with care [1]. Proper handling of lymph tracts can prevent damage and leakage of lymph fluids. Similarly, Inoue et al. described that an anastomosis onto the external iliac artery was a risk factor for SL [19]. They stated that the lymphatic fluid originates from the recipient's iliac lymph trunk rather than from the graft kidney. From an anatomical point of view, the lymph tracts around the iliac vessels are part of the lymphatic system of the pelvis. They collect lymph of the genital and urinary organs as well as that of the digestive tract. This system is composed of lymphatic nodes and vessels situated inside the conjunctive tissue, near the organs but especially along the external, internal and common iliac vessels [20,21]. We, therefore, advise to take great care regarding lymph tracts during dissection along the iliac vessels.

A second predictor for SL was related to the presence of a PD catheter at the time of surgery as well as its subsequent removal, whereas removal of the PD catheter when it was located at the same side of the abdomen as the fossa used for transplantation as well as opening the peritoneum reduced the risk for SL. We are not able to explain why a PD catheter located at the same side as the kidney transplantation would be a protecting factor for the development of 
SL. PD catheters are often inserted from the midline of the abdomen into cavum Douglasi and subsequently tunnelled to the right or left side of the abdomen. Nevertheless, these findings point to an important role of the peritoneum in the development of SLs. It is well known that peritoneal dialysis changes the composition of the peritoneum. Opening the peritoneum by fenestration is a well-known treatment for symptomatic lymphoceles [22-25]. Intentional fenestration might be an option to prevent SLs when it is safe to remove the PD catheter, at the risk of introducing a possibility of internal herniation of the intestine. The role of the peritoneum in the development of SLs may be a factor to assess in a future prospective study.

Another predictor for SLs was the presence of perfusion defects of the kidney after anastomosis and reperfusion, subsequently followed by partial ischaemia. Ischaemia and reperfusion affect many regulatory systems in the renal tissue and may trigger an immune response, resulting in a distinct inflammatory reaction of the kidney graft [26, 27]. This inflammation in turn leads to the attraction of more lymph fluid, causing accumulation of the lymph fluid around the kidney [28].

Another surgical predictor for SLs was the presence of a splint for more than 7 days and the use of a double $J$ catheter (21 days in situ) when compared to standard 5-day splinting. Our theory is that a plastic or silicone tube leads to irritation of the graft ureter. This irritation may then in turn lead to an inflammatory reaction resulting in an accumulation of lymph fluid and hence more symptomatic lymphoceles [29-31]. Irrespective of the type of tubing, splint or double $\mathrm{J}$ catheter, the duration seems to be the key factor. This finding is supported by the observation that the double $\mathrm{J}$ catheter was introduced in 2017; the same year a significant increase in SL was noted (14.7\% double J vs. $7.0 \%$ splint). We searched the literature to find a relation between type of stenting and its duration versus SL, but no reports were found. The wide variety in the reported incidences for SL might be related to the postoperative management of the graft ureter and ureteroneocystostomy.

This study showed that drain production was inversely related to the development of SLs. Apparently, less drain production leads to more SLs. Our hypothesis is that improper drainage of the retroperitoneal space directly after the kidney transplantation not only leads to accumulation of the non-drained blood and lymph fluid, but also facilitates more lymph fluid production as reaction to it and thus leads to an SL. This idea is supported by the finding that less SLs were seen in patients requiring a second operation for a postoperative bleeding, wherein blood is removed from the retroperitoneal space, fluid leakages are managed and a drain is properly (re-) replaced. We also investigated the effect of a thin versus a thick drain, but no significantly differences in the outcome of SLs were found. We advocate proper drain placement, advise to keep the drain open and to not remove the drain too quickly to prevent possible formation of an SL.

The slight increase in incidence of SLs over time might be attributed by the use of a double $\mathrm{J}$ as described earlier. Since the use of a double $\mathrm{J}$ was effectuated from 2017, this partly explains the increase. We, therefore, investigated other parameters that changed in time. A smaller drain diameter was used by May 2014, but no significant difference in SL formation was seen between the period prior and after the introduction. The same holds for the introduction of basiliximab as induction therapy starting July 2014. The trend towards more SLs is, therefore, unaccounted for.

\section{Limitations}

This is a retrospective study, which constitutes an important limitation. However, we obtained complete and detailed information from our database covering multiple years. Another limitation is the fact that these results are based on a single-centre study. Further research must show whether this applies to other settings as well.

Furthermore, since our group of patients that developed a symptomatic lymphocele was relatively small, i.e. out of 1003 patients only 45 did develop a symptomatic lymphocele, we were unable to do an extensive multivariate analysis.

\section{Conclusion}

This study showed that symptomatic lymphoceles develop in one out of 25 patients $(4.5 \%)$ receiving a kidney transplant and cause significant morbidity and mortality.

We found multiple surgical and non-surgical predictors for the development of a symptomatic lymphocele. The common denominators are the surgical management of the retroperitoneal space and peritoneum, management of the PD catheter at the moment of transplantation, wound drain production and the duration of stenting of the ureteroneocystostomy.

A prospective study is needed to confirm the role of these risk factors in the development of symptomatic lymphoceles. This study may lead to a better awareness and hopefully prevention of development of symptomatic lymphoceles in the future.

Funding All authors participated in research design, participated in writing of the paper, participated in the performance of the research and participated in data analysis. The authors declare no conflict of interest. No funding was received. The results presented in this paper have not been published previously in whole or part, except in abstract form. 


\section{Compliance with ethical standards}

Conflict of interest The authors declare no conflict of interest. This manuscript has been reviewed and approved by all of the authors, and no results reported in this manuscript have been published elsewhere. No funding was received. This article does not contain any studies with human participants or animals performed by any of the authors.

Open Access This article is distributed under the terms of the Creative Commons Attribution 4.0 International License (http://creativeco mmons.org/licenses/by/4.0/), which permits unrestricted use, distribution, and reproduction in any medium, provided you give appropriate credit to the original author(s) and the source, provide a link to the Creative Commons license, and indicate if changes were made.

\section{References}

1. Ranghino A, Segoloni GP, Lasaponara F et al (2015) Lymphatic disorders after renal transplantation: new insights for an old complication. Clin Kidney J 8(5):615-622

2. Zietek Z, Sulikowski T, Tejchman K et al (2007) Lymphocele after kidney transplantation. Transplant Proc 39:2744-2747

3. Goel M, Flechner SM, Zhou L et al (2004) The influence of various maintenance immunosuppressive drugs on lymphocele formation and treatment after kidney transplantation. J Urol 171:1788-1792

4. Adani GL, Baccarani U, Bresadola V et al (2005) Graft loss due to percutaneous sclerotherapy of a lymphocele using acetic acid after renal transplantation. Cardiovasc Intervent Radiol 28:836-838

5. Ulrich F, Niedzwiecki S, Fikatas P et al (2010) Symptomatic lymphoceles after kidney transplantation-multivariate analysis of risk factors and outcome after laparoscopic fenestration. Clin Transplant 24:273-280

6. Lucewicz A, Wong G, Lam VW et al (2011) Management of primary symptomatic lymphocele after kidney transplantation: a systematic review. Transplantation 92:663-673

7. Minetti EE (2011) Lymphocele after renal transplantation, a medical complication? J Nephrol 24:707-716

8. Gharbi C, Gueutin V, Izzedine H (2014) Oedema, solid organ transplantation and mammalian target of rapamycin inhibitor/ proliferation signal inhibitors (mTOR-I/PSIs). Clin Kidney J 7(2):115-120

9. Ebadzadeh MR, Tavakkoli M (2008) Lymphocele after kidney transplantation: where are we standing now? Urol J. 5(3):144-148

10. Bailey SH, Mone MC, Holman JM et al (2003) Laparoscopic treatment of post renal transplant lymphoceles. Surg Endosc 17(12):1896-1899

11. Fuller TF, Kang SM, Hirose R et al (2003) Management of lymphoceles after renal transplantation: laparoscopic versus open drainage. J Urol 169(6):2022-2025

12. Lucewicz A, Wong G, Lam VW et al (2011) Management of primary symptomatic lymphocele after kidney transplantation: a systematic review. Transplantation 92(6):663-673

13. Khauli RB, Stoff JS, Lovewell T et al (1993) Post-transplant lymphoceles: a critical look into the risk factors, pathophysiology and management. J Urol 150(1):22-26

14. Heer MK, Clark D, Trevillian PR et al (2018) Functional significance and risk factors for lymphocele formation after renal transplantation. ANZ J Surg 88(6):597-602
15. Inoue T, Saito M, Narita S et al (2017) Evaluation of persistent lymphatic fluid leakage using a strategy of placing a drain after kidney transplantation: a statistical analysis to assess its origin. Transplant Proc 49(8): 1786-1790

16. Thomas C, Ziewers S, Thomas A et al (2019) Development of symptomatic lymphoceles after radical prostatectomy and pelvic lymph node dissection is independent of surgical approach: a single-center analysis. Int Urology and Nephrology 51(4):633-640

17. Ebadzadeh M, Tavakkoli M (2008) Lymphocele after kidney transplantation: where are we standing now? Urol J 5(3):144-148

18. Martínez-Ocaña JC, Lauzurica R, Castellote E et al (1995) Adult polycystic kidney disease: a risk factor for lymphocele formation after renal transplantation? Transplant Proc 27(4):2246-2247

19. Inoue Saito, Narita et al (2017) Evaluation of persistent lymphatic fluid leakage using a strategy of placing a drain after kidney transplantation: a statistical analysis to assess its origin. Transplant Proc 49(8): 1786-1790

20. Wolfram-Gabel R (2013) Anatomy of the pelvic lymphatic system. Cancer Radiother 17(5-6):549-552

21. Assouad J, Riquet M, Foucault C et al (2006) Renal lymphatic drainage and thoracic duct connections: implications for cancer spread. Lymphology 39(1):26-32

22. Khauli RB, Mosenthal AC, Caushaj PF (1992) Treatment of lymphocele and lymphatic fistula following renal transplantation by laparoscopic peritoneal window. J Urol 147(5):1353-1355

23. Mihaljevic AL, Heger P, Abbasi Dezfouli S et al (2017) Prophylaxis of lymphocele formation after kidney transplantation via peritoneal fenestration: a systematic review. Transpl Int 30(6):543-555

24. Lucewicz A, Wong G, Lam VW et al (2011) Management of primary symptomatic lymphocele after kidney transplantation: a systematic review. Transplantation 92(6):663-673

25. Bailey SH, Mone MC, Holman JM et al (2003) Laparoscopic treatment of post renal transplant lymphoceles. Surg Endosc 17(12):1896-1899

26. Salvadori M, Rosso G, Bertoni E (2015) Update on ischemiareperfusion injury in kidney transplantation: pathogenesis and treatment. World J Transplant 5(2):52-67

27. Gu L, Tao Y, Chen C et al (2018) Initiation of the inflammatory response after renal ischemia/reperfusion injury during renal transplantation. Int Urol Nephrol 50(11):2027-2035

28. Sakai Y, Kobayashi M (2015) Lymphocyte 'homing' and chronic inflammation. Pathol Int 65(7):344-354

29. Wingate JT, Brandenberger J, Weiss A et al (2017) Ureteral stent duration and the risk of $\mathrm{BK}$ polyomavirus viremia or bacteriuria after kidney transplantation. Transpl Infect Dis 19(1):e12644. https://doi.org/10.1111/tid.12644

30. Lasaponara F, Dalmasso E, Santià S et al (2013) A 8-year-forgotten ureteral stent after kidney transplantation: treatment and long-term follow-up. Urologia 80(1):80-82

31. Lee JR, Bang H, Dadhania D, Hartono C et al (2013) Independent risk factors for urinary tract infection and for subsequent bacteremia or acute cellular rejection: a single-center report of 1166 kidney allograft recipients. Transplantation 96(8):732-738

Publisher's Note Springer Nature remains neutral with regard to jurisdictional claims in published maps and institutional affiliations. 